\title{
CALIBRATION OF DENSITOMETRY IN RADIO-ISOTOPIC IN SITU HYBRIDIZATION
}

\author{
JAN M RuiJTER, JACO HAgOORT, PIET AJ DE BOER AND ANTOON FM MOORMAN \\ Dept of Anatomy and Embryology, Academic Medical Centre, Meibergdreef 15, 1105 AZ, Amsterdam, the \\ Netherlands \\ e-mail: j.m.ruijter@amc.uva.nl \\ (Accepted August 28, 2001)
}

\begin{abstract}
Densitometry on autoradiographs of sections processed for in situ hybridization provides a direct measure for the in situ quantification of mRNA. Gelatin spots, containing different concentrations of the radioisotope, and processed in parallel with the tissue sections, can be used as a sensitive model to calibrate the densitometric measurements. The shape of the gelatin spots was shown to be circular with a parabolic crosssectional profile. This simple shape allows the subdivision of the spot into a series of concentric rings, which enables an unbiased measurement of the optical density - radioactivity relation. This spot measurement is also applicable to DNA arrays spotted on glass or membranes. A new model, explaining the optical density of autoradiographs, was derived and fitted to the calibration points. The use of this calibration method is crucial for the correct interpretation of autoradiographs
\end{abstract}

Keywords: calibration, densitometry, quantitative autoradiography.

\section{INTRODUCTION}

With the advent of molecular biology, the visualization of bio-molecules like mRNA with gelelectrophoresis has become quite fashionable, although by applying this procedure all histological information is lost. In situ hybridization with $\left[{ }^{35} \mathrm{~S}\right]$-labeled riboprobes on frozen or paraffin sections, followed by autoradiography, represents the most sensitive strategy for the in situ quantification of mRNA (Jonker et al., 1997; Moorman et al., 2000). Recently, phosphor screen imagers have been introduced into the field of histology (Vizi and Gulya, 2000; Ito et al., 1995), but the resolution required for detailed morphological studies cannot be reached without optical magnification and, therefore, autoradiograms are still indispensable. Unfortunately, it is still common practice to judge the staining intensity in sections with the naked eye, which can hardly be objectified because of the varying contrast within the sections (Shapley, 1986). Nowadays, the use of high resolution CCD cameras for image acquisition, has rendered one of the major objections against cytometry, the occurrence of a distributional error, obsolete: this error can either be considered to be irrelevant because the object size on the pixel of the camera is smaller then the optical resolution of the image acquisition system (Checo et al., 1994) and/or this error does not occur at all because silver grains in autoradiograms are fully opaque and, therefore, the transmittance is always equal to the fraction of coverage by grains (Jonker et al., 1997; Moorman et al., 2000). The use of bright field optical density (OD) measurements as a replacement for laborious grain counting has been validated for low radioactivity levels (Schnitzer et al., 1987). OD measurements were also shown to correlate well with tissue concentration of substances at higher radioactivity levels (Jonker et al., 1997). Also when a study only aims at relative quantification (Rogers, 1961) a calibration curve is indispensable because the relation between bound radioactivity and observed optical density can only be assumed to be linear for low activities (Swillens et al., 1989). The use of plastic calibration standards (Ito et al., 1995) has the disadvantage that the radioisotopes as well as the quenching conditions differ between standards and biological material. The same holds for dot blot standards on membranes (Palfi et al., 1998; Vizi and Gulya, 2000), which were introduced to avoid the efforts involved in preparing cryo-sectioned tissue paste standards (Miller, 1988) or embedded and sectioned gelatin standards (Schnitzer et al., 1987). Calibration spots on glass slides can be treated in parallel with the tissue sections and thus a calibration curve with the same radio-isotope can be obtained with the same quenching, tissue processing, and image 
acquisition conditions as the tissue sections (Larsson and Hougaard, 1994; Jonker et al., 1997). The local optical density (OD) of these calibration spots is related to the radioactivity per area unit. However, OD cannot increase indefinitely and the resulting relation between radioactivity and OD will be nonlinear. When the whole calibration spot, with a range from low to high OD values is measured at once, the mean OD will be averaged over this non-linear relation and, therefore, this mean will be biased downward. The resulting calibration curve will then lead to a misinterpretation of high OD values. A similar bias may occur with other quantification methods using spotted fluids like DNA arrays spotted on glass or membrane. The aim of the current study was to resolve this bias in the measurement of calibration spots and to determine procedural variables affecting the shape of the calibration curve.

\section{MATERIALS AND METHODS}

Gelatin calibration slides were prepared as described (Moorman et al., 2000). In short, a $4 \mu \mathrm{l}$ drop of a 5\% high gel-strength gelatin (48724, Fluka, Bachs, Switzerland) solution, containing one of 10 different concentrations of $\left[{ }^{35} \mathrm{~S}\right]$ - labeled cDNA was applied evenly over a circle (7 $\mathrm{mm}$ diameter) on microscope slides. The slides were dried and postfixed to prevent swelling of the spots later in the procedure. Samples of each $\left[{ }^{35} \mathrm{~S}\right]$-calibration solution were used for liquid scintillation counting to determine the amount of radioactivity applied on a spot. The autoradiographic procedure was carried out as described in detail (Moorman et al., 2000). Briefly, the slides were dipped in a photographic emulsion (Ilford Nuclear Research Emulsion G-5; diluted in water $1: 1.5 \mathrm{v} / \mathrm{v}$; two different batches), exposed for 3 to 14 days (Table 1), developed for 4 or $8 \mathrm{~min}$ and fixed. Two different batches of emulsion were used. Note that the initial activity of the spots decreased due to radioactive decay. A separate series of gelatin spots, without radioactivity but with a Blue Dextran stain, was prepared to determine the 3-dimensional shape of the gelatin spots. A peltier-cooled digital
CCD camera (Photometrics; $1317 \times 1035$ pixels; 12 bit; linear over its whole dynamic range) attached to a Zeiss Axiophot microscope, with normal bright field illumination was used for image acquisition. A stabilized light source, a $580 \mathrm{~nm}$ band pass filter, a $400-800 \mathrm{~nm}$ infrared block filter and a $1.25 \mathrm{x}$ magnification were used. After focusing on the object, an image of the incident light $\left(I_{0}\right)$ was recorded without a slide on the stage. Subsequently, without changing illumination, magnification or shutter time, for each spot an image $\left(I_{i}\right)$ was recorded and converted into an optical density (OD) image using $\boldsymbol{O} \boldsymbol{D}_{\boldsymbol{i}(x, y)}=-{ }^{10} \log \left(I_{i(x, y)} / I_{0(x, y)}\right)$ (Oberholzer et al., 1996). This conversion incorporates implicitly an illumination-shading correction (Chieco et al., 1994). Images were binned to $658 \times 517$ pixels, which reduces photon noise, and stored as 8 bit images (pixel value = $250 \times$ OD). Image acquisition and conversion were carried out with PMIS (www.gkrc.com; version 4.1). For densitometry the public domain image analysis program NIH Image was used (rsb.info.nih.gov/nihimage; version 1.61). Curve fitting and testing of differences between groups were done with the least squares non-linear regression and analysis of variance procedures of SPSS (SPSS Inc; release 10.0.7).

\section{RESULTS AND DISCUSSION}

\section{3-DIMENSIONAL SHAPE OF THE GELATIN SPOTS}

A series of Blue Dextran-stained gelatin spots was used to determine the 3-dimensional shape of the spots. Staining intensity of these spots was such that a linear relation between OD and spot height existed. Measurement of the length of two orthogonal crosssections showed on average no difference between the two cross-sections (Fig. 1A). The pixel value profile of the spots could be fitted to a parabola $\left(y=a+c x^{2}\right)$ in all cases although a wide range of heights $(a)$ and base lengths (diameter $=2 \sqrt{ }(-a / c)$ ) were observed (Fig. 1B). From these results it can be concluded that the 3D shape of the gelatin calibration spots used in this study is circular with a parabolic cross-sectional profile. 
Table 1. Material used for determining effects of exposure time, development time and emulsion on the optical density.

\begin{tabular}{cccc}
\hline initial activity & exposure time (days) & development time (min) & batch of emulsion \\
\hline 100 & 6 & 4 & 1 \\
98 & 3 & 4 & 1 \\
90 & 6 & 4 & 1 \\
46 & 6 & 8 & 2 \\
46 & 6 & 4 & 2 \\
46 & 7 & 4 & 2 \\
46 & 14 & 4 & 2 \\
\hline
\end{tabular}

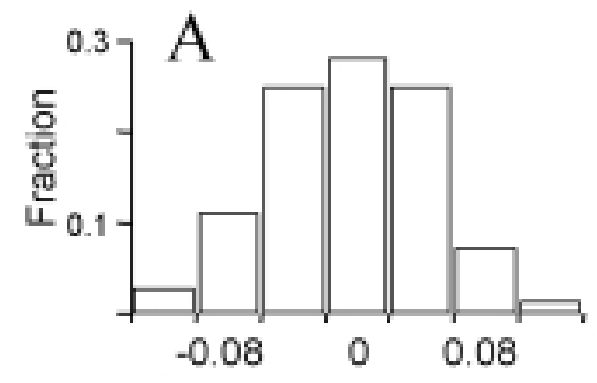

$(\mathrm{L}-\mathrm{R}) /(\mathrm{L}+\mathrm{R}) / 2\}$

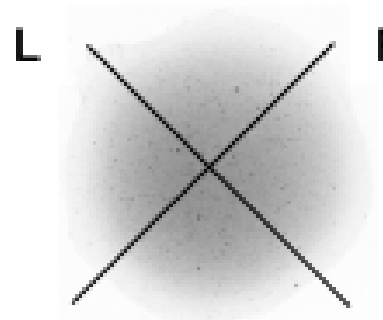

$\mathbf{R}$

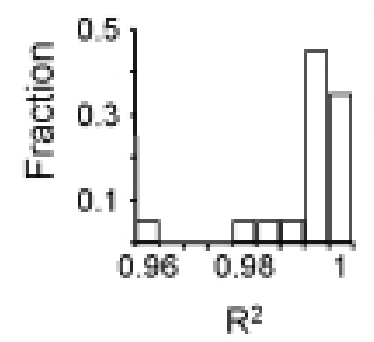

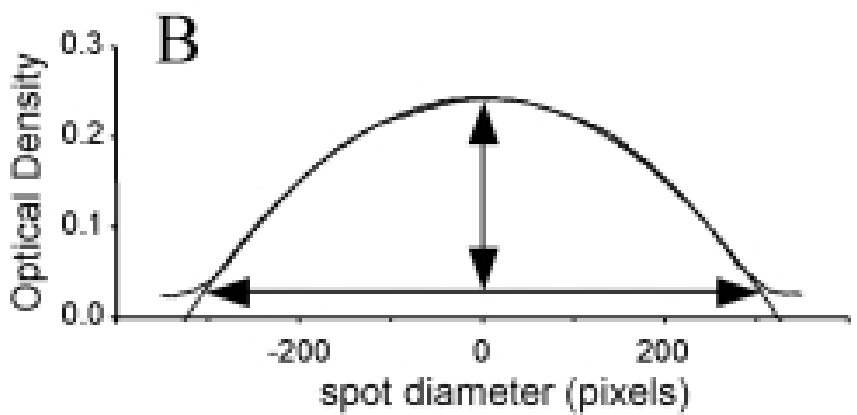

spot diameter (pixels)
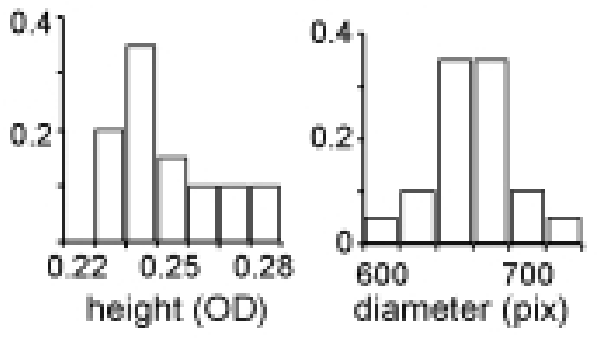

Fig. 1. Shape of the gelatin calibration spots. A. Gelatin spot with two orthogonal profiles and frequency distribution of the difference in profile length. $\boldsymbol{B}$. Fit of a parabola to the cross-sectional profile of a gelatin spot with frequency distributions of the correlation coefficient $\left(R^{2}\right)$, height and diameter.

\section{DERIVATION OF THE FRACTIONAL VOLUME OF CONCENTRIC RINGS}

To avoid OD measurements over a range of OD values, the calibration spots have to be measured in a series of concentric rings and the volume per ring must be determined. The total volume of a parabolic spot is $\pi c^{2} / 2 a$, and the volume of the ring $i$ bound by $\mathrm{X}_{\mathrm{i}}$ and $\mathrm{X}_{\mathrm{i}+1}$ is $\pi\left(Y_{i}^{2}+Y_{i+1}^{2}\right) / 2 a$, which leads to a volume fraction of this ring of $\left(Y_{i}^{2}+Y_{i+1}{ }^{2}\right) / c^{2}$. When the spot is divided into $n$ concentric rings, the latter equation can be rearranged into:

$$
\text { Volume Fraction }_{i}=\frac{\left(i^{2}-n^{2}\right)^{2}-\left\{(i+1)^{2}-n^{2}\right\}^{2}}{n^{4}} .
$$

Eq. 1 shows that the volume fraction of ring $i$ ( $i$ from 0 to $n-1)$ is only dependent on the number of rings $(n)$ and the ring number $(i)$; the parabola parameters $a$ and $c$, and therefore the height and the diameter of the spot, are cancelled out. Fig. 2 illustrates how the volume of a parabolic spot is distributed over concentric rings. 


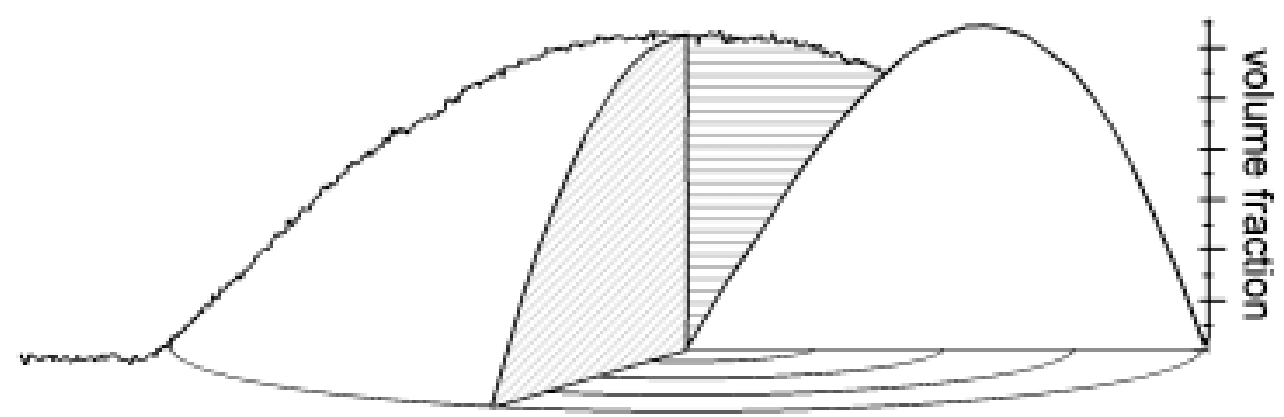

Fig. 2. A gelatin calibration spot has a circular base with a parabolic cross-section. The graph shows the distribution of the volume of the spot over concentric rings.

\section{MEASUREMENT OF CALIBRATION SPOTS WITH CONCENTRIC RINGS}

The simple relation between rings and volume fraction allowed the measurement of the spots by subdividing them into a series of concentric rings. Two distinct situations occurred, depending on the microscopic magnification. When spots could be fully contained in the field of the camera, concentric regions were constructed through binarization of the whole spot, a distance transformation from the outer contour inward (in_dist) and a distance transformation from the center outwards (out_dist). The relative position of each pixel in the spot can then be calculated as out_dist/(out_dist + in_dist $)$. Ranges of relative position values give concentric 'rings', irrespective of small deviations from the circular outline of the spot. By taking these ranges of relative positions, concentric regions of the original spot can be masked and their area and mean OD can be measured. When spots are not completely present in the image, the average diameter of the spots has to be determined by measuring the length of two orthogonal cross-sections. Then a series of concentric rings with increasing diameter is superimposed on the center of the spot and the area and mean OD in these rings can be measured. For subsequent calculation of the volume fraction of each concentric ring or region, the cumulative area with and without the ring is used to calculate the inner and outer radius of the ring. These radii and the radius of the total spot, calculated from the total area or measured from the cross-section, can then be used to calculate the volume fraction of the ring, using the relation between rings and volume (Eq. 1). From this volume fraction, the total radioactivity of the spot and the ring area the radioactivity, expressed as counts per minute (CPM) per pixel can be calculated. Both methods of measurement result in the same relation between OD and radioactivity per pixel, which means that not the whole calibration spot needs to be measured, as long as the diameter can be determined. The curve of both methods differs significantly from the relation that was found when the mean OD of the whole spot is measured (Fig. 3). The dynamic range of the calibration curve is extended two-fold by measuring concentric rings.

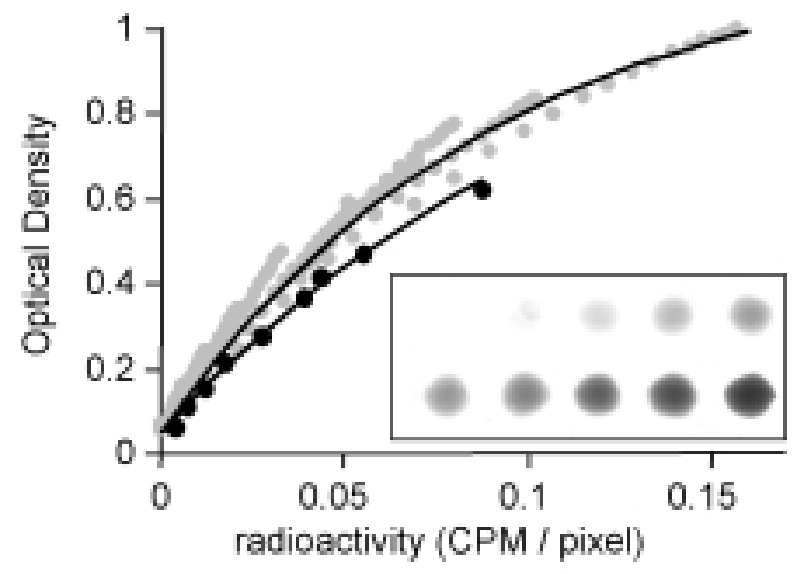

Fig. 3. Results of the measurement of mean $O D$ of the 10 whole spots (black dots) and measurement of 15 concentric rings on each spot (gray dots). Solid lines are fitted with Eq. 2. Inset: calibration slide.

\section{RELATION BETWEEN RADIOACTIVITY AND OPTICAL DENSITY}

Recently, Palfi and co-workers (1998) derived a mathematical model for the optical density of autoradiographs in which they incorporated the radioactivity $(\mathrm{X})$ and two factors depending on the film emulsion: $O D=p_{1}\left(1-e^{-p_{2} \cdot X}\right)$. In this formula $\mathrm{p}_{1}$ depends on silver ion concentration and development time and $\mathrm{p}_{2}$ on radioisotope and exposure time. In photography, the relation between OD and development time is known to be: $O D=O D_{\max }\left(1-e^{-f . t}\right)$, with $O D_{\max }$ and $f$ as emulsion dependent maximum density and 
development constant, respectively, and $t$ as development time. With these equations and the formula for autoradiographic grain density derived by Perry (1964), we derived a formula that incorporates development time, exposure time and radioactivity as variables and three emulsion dependent constants $\left(O D_{\max }\right.$, development constant $f$ and exposure constant $b$ ):

$$
\begin{gathered}
\mathbf{O D}=\text { background }+\mathrm{OD}_{\max } \cdot \\
\left(1-\mathrm{e}^{-\mathrm{f} . \text { Dev_time }}\right) \cdot\left(1-\mathrm{e}^{-\mathrm{b} . \text { Exp_time } \cdot \text { radioactivity }}\right),
\end{gathered}
$$

in which background is the OD of the glass slide. Note that because of the multiple products in the equation the curve fitting is very sensitive to starting values.

\section{EFFECT OF EMULSION, DEVELOPMENT TIME, AND EXPOSURE TIME}

Various series of calibration spots (Table 1) were measured to determine the influence of emulsion, exposure time and development time on the relation between OD and radioactivity. For all series the correlation coefficient of the fit to Eq. 2 was better than 0.95 . The background and $O D_{\max }$ parameters were 0.05 and 2.0 , respectively, and were the same for both emulsions whereas the development factor $f$ was found to be constant per emulsion batch ( 0.2 and 0.14, respectively). Comparison of series with different development times (Fig. 4B) showed that with the value of $f$ for the second emulsion, the difference in development time fully explained the difference in OD. A longer exposure time leads to higher OD values (Fig. 4A). However, when series with the same development time but exposure times of 3 and 6 days were compared it appeared that the same dose (= exposure time $\mathrm{X}$ radioactivity) resulted in higher OD values when it was applied over a longer time period (Fig. 4A, inset). A similar effect was found for exposure times of 7 and 14 days. This exposure time dependence was reflected in the values of the exposure factor $b(0.0015$ and 0.00115 , emulsion 1 for 6 and 3 days of exposure, respectively). The value of the exposure factor was also found to depend on the dilution of the emulsion: in another aliquot of emulsion 1 the value of $b$ is decreased from 0.0015 to 0.0009 . Because of this variability each series of tissue sections need to be accompanied by a parallel-treated calibration slide.

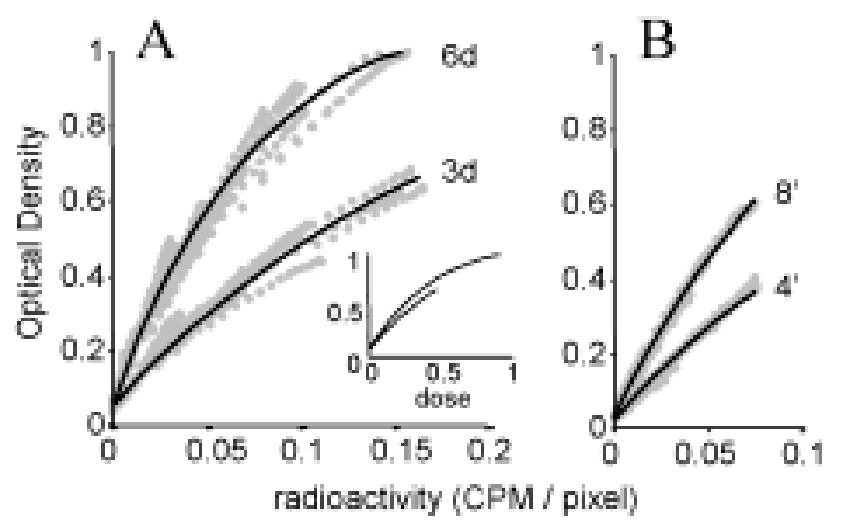

Fig. 4. A. Effect of exposure time (3 or 6 days) on the radioactivity - $O D$ relation. Inset: same data with dose (= radioactivity $\times$ exposure time) as $X$-axis. $\boldsymbol{B}$. Effect of development time (4 or $8 \mathrm{~min}$ ) on the radioactivity $-O D$ relation.

\section{SPOT VARIABILITY AND EMULSION THICKNESS}

In a separate series of calibration slides the reproducibility of the calibration spots and the effect of the emulsion thickness were determined. On slides that were dipped in the emulsion for one, two, or three times, a significant increase in OD of the spots was observed but not of the background staining of the slide. This shows that a thicker emulsion results in a better signal to noise ratio. No differences were found between spots on different positions on the slide or between spots on different slides indicating the absence of significant differences in emulsion thickness within and between slides.

\section{CONCLUDING REMARKS}

The present results show that the measurement of calibration spots as a series of concentric rings fully corrects the bias caused by the non-linear relation between radioactivity and OD. This method is also applicable to spotted DNA samples used in macro and micro arrays on glass or membrane. For proper interpretation of autoradiographs conversion from OD values to radioactivity values should always precede the comparison of mRNA levels in tissues. Because of the shape of the calibration curve the OD values cannot be reliably used to determine absolute or relative differences between tissues. In conclusion, the presented methods for the construction and fitting of a calibration curve greatly enhance the sensitivity of the quantification of autoradiography. 


\section{ACKNOWLEDGEMENT}

The authors were supported by the Netherlands Heart Foundation (NHF-grant 96.002).

\section{REFERENCES}

Chieco P, Jonker A, Melchiorri C, Vanni G, van Noorden CJF (1994). A user's guide for avoiding errors in absorbance image cytometry: a review with original experimental observations. Histochemical Journal 26:1-19.

Ito T, Suzuki T, Lim DK, Wellman SE Ho IK (1995). A novel quantitative receptor autoradiography and in situ hybridization histochemistry technique using storage phosphor screen imaging. J Neurosci Methods 59:265-71.

Jonker A, de Boer PAJ, van den Hoff MJ, Lamers WH, Moorman AF (1997). Towards quantitative in situ hybridization. J Histochem Cytochem 45:413-23.

Larsson L-I, Hougaard DM (1994). Glass slide models for immunocytochemistry and in situ hybridization. Histochemistry 101:325-31.

Miller JA (1988). An improved calibration procedure for computer-based quantitative autoradiography utilizing a mathematical model for non-linear response of camera and film. J Neurosci Methods 22:233-8.

Moorman AFM, De Boer PAJ, Ruijter JM, Hagoort J, Franco D, Lamers WH (2000). Radio-isotopic in situ hybridization on tissue sections: practical aspects and quantification. In: Tuan RS, Lo CW, eds. Methods in Molecular Biology, Vol. 137: Developmental Biology
Protocols, Vol. III, Ch 11. Totowa, New York: Humana Press Inc.

Oberholzer M, Östreicher M, Christen H, Brühlmann M (1996). Methods in quantitative image analysis. Histochem Cell Biol 105:333-55.

Palfi A, Hatvani L, Gulya K (1998). A new quantitative film autoradiographic method of quantifying mRNA transcripts for in situ hybridization. J Histochem Cytochem 46:1141-9.

Perry RP (1964). Quantitative autoradiography. In: Presscot DM ed. Methods in cell physiology. Ch 15. New York: Academic Press, 305-26.

Rogers AW (1961). A simple photometric device for the quantitation of silver grains in autoradiographs of tissue sections. Exp Cell Res 24:228-39.

Shapley R (1986). The importance of contrast for the activity of single neurons, the VEP and perception. Vision Res 26:45-61.

Schnitzer JJ, Morrel EC, Colton CK, Smith KA, Stemerman MB (1987). Absolute quantitative autoradiography of low concentrations of $\left[{ }^{125} \mathrm{I}\right]$-labeled proteins in arterial tissue. J Histochem Cytochem 35:1439-50.

Swillens S, Cochaux P, Lecocq R (1989). A pitfall in computer-aided quantification of autoradiograms. Trends Biochem Sci 14:440-1.

Vizi S, Gulya K (2000). Calculation of maximal hybridization capacity (Hmax) for quantitative in situ hybridization: a case study for multiple calmodulin mRNAs. J Histochem Cytochem 48:893-904. 